\title{
Novel Thienopyrimidine Derivatives Containing 1,2,4-triazoles and 1,3,4-oxadiazoles as Potent Antimicrobial Activity
}

Nagaraju Kerru, Triloknadh Settypalli, Harikrishana Nallapaneni and Venkata Rao Chunduri*

Department of Chemistry, Sri Venkateswara University, Tirupati 517 502, Andhra Pradesh, India

\begin{abstract}
A series of thienopyrimidine oxadiazole derivatives (5a-l) were synthesized by cyclic condensation of substituted aroylhydrazides with triazole of carboxylic acid $(4 \mathrm{a}-\mathrm{c})$. The synthesized compounds were characterized on the basis of their spectral (IR, ${ }^{1} \mathrm{H}$ and ${ }^{13} \mathrm{C}$ NMR) data and screened for antimicrobial activity where compounds $5 \mathrm{~b}$ and $5 \mathrm{~d}$ showed significant activity.
\end{abstract}

Keywords: Thienopyrimidine; 1,2,4-triazole; 1,3,4-oxadiazole; Antibacterial; Antifungal activity

\section{Introduction}

The firm appearances of microbial infections followed by the spreading out of numerous resistant bacterial and fungal strains against clinically used antimicrobial have insist on medicinal communities to look for new incorporations into the current methods used in medicine. Severe probability of microbial infections along with immunosuppressive individuals due to the HIV infection, cancer treatments and organ transplantations actuated additional urgency to generate new antimicrobial agents. Moreover, in some cases especially in patients with impaired liver or kidney functions bring into play of antimicrobial drugs to pleasure infections causes several problems [1-3]. Thus, these trends have emphasized the urgent, innovative more helpful and safe antimicrobial agents. Along with the striking approaches to attain this goal the development of structurally new classes of antimicrobial agents with novel mechanism of action and the other contained structural modification or optimization of the existing agents by improving both the binding affinity and spectrum of activity while retaining bioavailability and safety profile have provoked special interest in the area of medical chemistry. However, the increasing dominance of one such strategy that has been pursued in recent years employs a combination of two different active fragments in one molecule has emerged [4]. With this strategy, each drug moiety is designed to bind independently two different biological targets and synchronously accumulate at mutually intention sites. Such twin exploit drugs or hybrid drugs suggest the possibility to overcome the current resistance and reduce the appearance of new resistant strains [5].

In the past few years, thienopyrimidines have concerned immense thought due to their impressive array of pharmacological activities. They were found to display antiviral [6], antimicrobial [7], antihypertensive [8], analgesic and anti-inflammatory activities [9]. Moreover, a literature survey revealed that novel thienopyrimidine derivatives which have been more recently studied not only antiviral and antimicrobial activities but also inhibit various protein kinase such as CK2 involved in particular anticancer activity [10]. In addition, the extensive utilize of 1,3,4-oxadiazoles as a scaffold in curative chemistry establishes this moiety as a member of the privileged structures class [11]. 1,3,4-oxadizole ring was associated with many types of biological properties such as anti inflammatory [12], insecticidal [13], antifungal, antibacterial and antituberculosis activities [14,15]. In this context, thienopyrimidine containing 1,2,4-triazole derivatives with similar structural qualities would be projected to result in newer molecular systems with increased efficacy. Definitely, 1,2,4-triazole template has been known to express considerable antimicrobial [16], antitubercular
[17] and anticancer activities [18]. In continuation to extend our research $[19,20]$ it was our thought of interest to design and synthesize thienopyrimidine 1,3,4-oxadiazole derivatives hoping to go a step forward in the field of antioxidant agents. Taking the above points in consideration, we have studied the antimicrobial action of the resultant thienopyrimidine 1,2,4-triazole and 1,3,4-oxadiazole derivatives against wide range of different microorganisms.

\section{Experimental}

\section{Chemistry}

Melting points were determined in open capillaries on a Mel-Temp apparatus and are uncorrected. All the reactions were monitored by thin layer chromatography (TLC) on precoated silica gel 60 F254 (mesh); spots were visualized with UV light. Merck silica gel (60-120 mesh) was used for column chromatography. The IR spectra were recorded on a Perkin-Elmer BX1 FTIR Spectrophotometer as $\mathrm{KBr}$ pellets and the wave numbers were given in $\mathrm{cm}^{-1} .{ }^{1} \mathrm{H}$ NMR $(400 \mathrm{MHz})$ and ${ }^{13} \mathrm{C}$ NMR $(100 \mathrm{MHz})$ spectra were recorded on a Bruker AMX $400 \mathrm{MHz}$ NMR spectrometer in $\mathrm{CDCl}_{3} / \mathrm{DMSO}-d_{6}$ solution using TMS as an internal standard. The mass spectra were recorded on Agilent $1100 \mathrm{LC} / \mathrm{MSD}$ instrument with method API-ES at $70 \mathrm{eV}$. All chemical shifts are reported in $\delta$ (ppm) using TMS as an internal standard.

Procedure for the preparation of ethyl 4-cyano-5( (ethoxymethylene) amino)-3-methylthiophene-2 carboxylate (2)

A mixture of ethyl 5-amino-4-cyano-3-methylthiophene-2carboxylate [21] $1(2.10 \mathrm{~g}, 10 \mathrm{mmol})$ in triethyl orthoformate $(12$ $\mathrm{mL}$ ) was heated under refluxion for $14 \mathrm{~h}$. After completion of starting compound, the excess amount of triethyl orthoformate was removed under vacuum. The residue was washed with petroleum ether then they obtain solid was filtered and recrystallized from DMSO water.

Yield: $89 \%$ (brown color solid); m.p. $236-238^{\circ} \mathrm{C}$; IR $\left(\mathrm{KBr}, \mathrm{cm}^{-1}\right)$ :

*Corresponding authors: Venkata Rao Chunduri, Department of Chemistry, Sr Venkateswara University, Tirupati 517502, Andhra Pradesh, India, Tel: +91-8772289303; Fax: +91-877-2249532; E-mail: cvrsvu@gmail.com

Received July 31, 2014; Accepted August 27, 2014; Published August 30, 2014

Citation: Kerru N, Settypalli T, Nallapaneni H, Chunduri VR (2014) Nove Thienopyrimidine Derivatives Containing 1,2,4-triazoles and 1,3,4-oxadiazoles as Potent Antimicrobial Activity. Med chem 4: 623-629. doi:10.4172/2161 0444.1000204

Copyright: $\odot 2014$ Kerru N, et al. This is an open-access article distributed under the terms of the Creative Commons Attribution License, which permits unrestricted use, distribution, and reproduction in any medium, provided the original author and source are credited. 
2852, 1715, 1622, 1545; ${ }^{1} \mathrm{H}$ NMR $\left(400 \mathrm{MHz} ; \mathrm{CDCl}_{3}\right): \delta_{\mathrm{H}} 1.37(\mathrm{t}, 3 \mathrm{H}$, $\left.-\mathrm{OCH}_{2} \mathrm{CH}_{3}\right), 1.43\left(\mathrm{t}, 3 \mathrm{H}, \mathrm{CH}_{3}\right.$-ester), $2.58\left(\mathrm{~s}, 3 \mathrm{H}, \mathrm{CH}_{3}\right), 4.32(\mathrm{q}, 2 \mathrm{H}$, $\left.-\underline{\mathrm{OCH}}_{2} \mathrm{CH}_{3}^{-}\right), 4.47\left(\mathrm{q}, 2 \mathrm{H}, \mathrm{CH}_{2}\right.$-ester), $7.90(\mathrm{~s}, 1 \mathrm{H}, \mathrm{CH}) ;{ }^{13} \mathrm{C} \mathrm{NMR}(100$ $\left.\mathrm{MHz} ; \mathrm{CDCl}_{3}\right): \delta_{\mathrm{C}} 12.33,16.41,19.37,60.73,65.26,112.64,116.08$, $121.17,141.32,151.50,157.86,161.91$; LC-MS $(70 \mathrm{eV}): \mathrm{m} / \mathrm{z}=267$ $(\mathrm{M}+\mathrm{H})^{+}$.

\section{General procedure for the synthesis of compound (3a-c)}

A mixture of $2(0.26 \mathrm{~g}, 1 \mathrm{mmol})$ and corresponding substituted aroylhydrazides $\left(\mathrm{R}-\mathrm{PhCONHNH}{ }_{2}\right)(1 \mathrm{mmol})$ in toluene $(10 \mathrm{~mL})$ was stirred at room temperature then glacial acetic acid $(0.1 \mathrm{mmol})$ was added and the reaction mixture was refluxed for $12 \mathrm{~h}$. After completion of the reaction, the solvent was removed under reduced pressure then it was washed with water to obtained solid, filtered and recrystallized from ethanol $[22,23]$.

Ethyl 3-(4-chlorophenyl)-9-methylthieno[3,2-e][1,2,4] triazolo[4,3-c]pyrimidine-8-carboxylate (3a)

Yield: $84 \%$ (pale brown color solid); m.p. $218-220^{\circ} \mathrm{C}$; IR (KBr, cm $\left.{ }^{1}\right): 2988,1705,1630,1538 ;{ }^{1} \mathrm{H} \mathrm{NMR}\left(400 \mathrm{MHz} ; \mathrm{CDCl}_{3}\right): \delta_{\mathrm{H}} 1.44(\mathrm{t}, 3 \mathrm{H}$, $\mathrm{CH}_{2}$-ester), $3.18\left(\mathrm{~s}, 3 \mathrm{H}, \mathrm{CH}_{3}\right), 4.44\left(\mathrm{q}, 2 \mathrm{H}, \mathrm{CH}_{3}\right.$-ester), $7.49\left(\mathrm{~d}, \mathrm{~J}_{\mathrm{HH}}=\right.$ $8.0 \mathrm{~Hz}, 2 \mathrm{H}, \mathrm{Ar}-\mathrm{H}), 8.29$ (d, $\left.J_{\mathrm{HH}}=8.0 \mathrm{~Hz}, 2 \mathrm{H}, \mathrm{Ar}-\mathrm{H}\right), 9.24(\mathrm{~s}, 1 \mathrm{H}, \mathrm{CH}-$ pyrimidine); ${ }^{13} \mathrm{C}$ NMR $\left(100 \mathrm{MHz} ; \mathrm{CDCl}_{3}\right): \delta_{\mathrm{C}} 9.37,20.68,62.15,121.92$, $124.03,128.11,129.62,131.67,134.75,139.94,142.26,147.47,156.28$, 159.43, 162.28; LC-MS $(70 \mathrm{eV}): \mathrm{m} / \mathrm{z}=373(\mathrm{M}+\mathrm{H})^{+}$.

Ethyl 9-methyl-3-phenylthieno[3,2-e][1,2,4] triazolo [4,3-c] pyrimidine-8-carboxylate (3b)

Yield: $81 \%$ (brown color solid); m.p. 202-204 ${ }^{\circ} \mathrm{C}$; IR $\left(\mathrm{KBr}, \mathrm{cm}^{-1}\right)$ : 2898, 1716, 1621, 1524; ${ }^{1} \mathrm{H}$ NMR $\left(400 \mathrm{MHz} ; \mathrm{CDCl}_{3}\right): \delta_{\mathrm{H}} 1.43(\mathrm{t}, 3 \mathrm{H}$, $\mathrm{CH}_{2}$-ester), 3.16 (s, $\left.3 \mathrm{H}, \mathrm{CH}_{3}\right), 4.42$ (q, $2 \mathrm{H}, \mathrm{CH}_{3}$-ester), 7.48-8.31 (m, $5 \mathrm{H}, \mathrm{Ar}-\mathrm{H}), 9.22$ (s, $1 \mathrm{H}, \mathrm{CH}-$ pyrimidine); ${ }^{13} \mathrm{C} \mathrm{NMR}\left(100 \mathrm{MHz} ; \mathrm{CDCl}_{3}\right.$ ): $\delta_{\mathrm{C}} 11.42,19.36,64.07,120.38,125.96,128.03,130.33,131.48,136.25$, $140.91,144.64,146.22,151.16,155.68,160.10$; LC-MS $(70 \mathrm{eV}): \mathrm{m} / \mathrm{z}=$ $339(\mathrm{M}+\mathrm{H})^{+}$.

Ethyl 9-methyl-3-(p-tolyl)thieno[3,2-e][1,2,4] triazolo[4,3-c] pyrimidine-8-carboxylate (3c)

Yield: $86 \%$ (yellow color solid); m.p. $210-212^{\circ} \mathrm{C}$; IR $\left(\mathrm{KBr}, \mathrm{cm}^{-1}\right)$ : 2851, 1706, 1632, 1562; ${ }^{1} \mathrm{H}$ NMR $\left(400 \mathrm{MHz} ; \mathrm{CDCl}_{3}\right): \delta_{\mathrm{H}} 1.36(\mathrm{t}, 3 \mathrm{H}$, $\mathrm{CH}_{2}$-ester), $2.43\left(\mathrm{~s}, 3 \mathrm{H}, \mathrm{CH}_{3}\right), 3.17\left(\mathrm{~s}, 3 \mathrm{H}, \mathrm{CH}_{3}\right), 4.43\left(\mathrm{q}, 2 \mathrm{H}, \mathrm{CH}_{3}-\right.$ ester), $7.29\left(\mathrm{~d}, J_{\mathrm{HH}}=12.0 \mathrm{~Hz}, 2 \mathrm{H}, \mathrm{Ar}-\mathrm{H}\right), 8.21\left(\mathrm{~d}, J_{\mathrm{HH}}=8.0 \mathrm{~Hz}, 2 \mathrm{H}\right.$, $\mathrm{Ar}-\mathrm{H}), 9.21$ (s, $1 \mathrm{H}, \mathrm{CH}$-pyrimidine); ${ }^{13} \mathrm{C} \mathrm{NMR}\left(100 \mathrm{MHz} ; \mathrm{CDCl}_{3}\right.$ ): $\delta_{C} 12.33,21.90,26.46,58.68,122.44,126.05,129.41,131.65,133.60$, $137.57,141.01,145.36,148.20,153.06,156.71,163.48$; LC-MS (70 eV): $\mathrm{m} / \mathrm{z}=353(\mathrm{M}+\mathrm{H})^{+}$.

\section{General procedure for the synthesis of compound (4a-c)}

The compounds (3a-c) were dissolved in $\mathrm{MeOH}(12 \mathrm{~mL})$ and $15 \% \mathrm{v} / \mathrm{v}$ aq. $\mathrm{NaOH}(2 \mathrm{~mL})$ was added and stirred for $16 \mathrm{~h}$ at room temperature. After completion of the reaction, chloroform was added to it then the aqueous layer was separated and acidified with $1 \mathrm{~N} \mathrm{HCl}$, the solid was filtered and washed with water, dried and recrystallized from chloroform and methanol.

3-(4-Chloro-phenyl)-9-methyl-thieno $[3,2$-e $][1,2,4]$ triazolo[4,3-c]pyrimidine-8-carboxylic acid (4a)

Yield: 78\% (yellow color solid); m.p. $224-226^{\circ} \mathrm{C}$; IR $\left(\mathrm{KBr}, \mathrm{cm}^{-1}\right)$ : 3356, 2862, 1702, 1641, 1539; ${ }^{1} \mathrm{H}$ NMR $\left(400 \mathrm{MHz} ; \mathrm{CDCl}_{3}\right): \delta_{\mathrm{H}} 2.01(\mathrm{~s}$, $\left.3 \mathrm{H}, \mathrm{CH}_{3}\right), 7.46\left(\mathrm{~d}, J_{\mathrm{HH}}=12.0 \mathrm{~Hz}, 2 \mathrm{H}, \mathrm{Ar}-\mathrm{H}\right), 7.68\left(\mathrm{~d}, J_{\mathrm{HH}}=8.0 \mathrm{~Hz}, 2 \mathrm{H}\right.$,
Ar-H), 8.86 (s, 1H, CH-pyrimidine), 10.14 (s, 1H, COOH); ${ }^{13} \mathrm{C}$ NMR $\left(100 \mathrm{MHz} ; \mathrm{CDCl}_{3}\right): \delta_{\mathrm{C}} 13.17,110.68,120.55,126.75,129.02,131.42$, $134.26,139.31,142.53,146.61,150.44,158.20,161.01$; LC-MS (70 eV): $\mathrm{m} / \mathrm{z}=345(\mathrm{M}+\mathrm{H})^{+}$.

\section{9-Methyl-3-phenyl-thieno[3,2-e] [1,2,4] triazolo[4,3-c] pyrimidine-8-carboxylic acid $(4 \mathrm{~b})$}

Yield: $80 \%$ (pale yellow color solid); m.p. $232-234^{\circ} \mathrm{C}$; IR $(\mathrm{KBr}, \mathrm{cm}$ $\left.{ }^{1}\right): 3364,2892,1711,1602,1512 ;{ }^{1} \mathrm{H} \mathrm{NMR}\left(400 \mathrm{MHz} ; \mathrm{CDCl}_{3}\right): \delta_{\mathrm{H}} 1.86(\mathrm{~s}$, $3 \mathrm{H}, \mathrm{CH}_{3}$ ), 7.23-7.41 (m, 5H, Ar-H), 8.90 (s, 1H, CH-pyrimidine), 10.32 (s, $1 \mathrm{H}, \mathrm{COOH}) ;{ }^{13} \mathrm{C} \mathrm{NMR}\left(100 \mathrm{MHz} ; \mathrm{CDCl}_{3}\right): \delta_{\mathrm{C}} 23.46,118.71,124.64$, $125.02,129.51,133.18,136.73,139.22,144.10,148.31,152.26,159.54$, 163.41; LC-MS $(70 \mathrm{eV}): \mathrm{m} / \mathrm{z}=311(\mathrm{M}+\mathrm{H})^{+}$.

\section{9-Methyl-3-p-tolyl-thieno[3,2-e] $[1,2,4]$ triazolo $[4,3-c]$ pyrimidine-8-carboxylic acid (4c)}

Yield: $81 \%$ (yellowish green color solid); mp 212-214 ${ }^{\circ} \mathrm{C}$; IR $(\mathrm{KBr}$, $\left.\mathrm{cm}^{-1}\right): 3319,2889,1714,1656,1542 ;{ }^{1} \mathrm{H}$ NMR $\left(400 \mathrm{MHz} ; \mathrm{CDCl}_{3}\right): \delta_{\mathrm{H}}$ $1.42\left(\mathrm{~s}, 3 \mathrm{H}, \mathrm{CH}_{3}\right), 2.68\left(\mathrm{~s}, 3 \mathrm{H}, \mathrm{CH}_{3}\right), 6.94\left(\mathrm{~d}, J_{\mathrm{HH}}=8.0 \mathrm{~Hz}, 2 \mathrm{H}, \mathrm{Ar}-\mathrm{H}\right)$, $7.12\left(\mathrm{~d}, J_{\mathrm{HH}}=8.0 \mathrm{~Hz}, 2 \mathrm{H}, \mathrm{Ar}-\mathrm{H}\right), 8.76(\mathrm{~s}, 1 \mathrm{H}, \mathrm{CH}$-pyrimidine $), 10.62$ $(\mathrm{s}, 1 \mathrm{H}, \mathrm{COOH}) ;{ }^{13} \mathrm{C} \mathrm{NMR}\left(100 \mathrm{MHz} ; \mathrm{CDCl}_{3}\right): \delta_{\mathrm{C}} 19.36,32.11,114.45$, $121.87,127.91,130.10,132.51,137.02,140.73,145.09,149.26,153.14$, 158.16, 164.23; LC-MS $(70 \mathrm{eV}): \mathrm{m} / \mathrm{z}=325(\mathrm{M}+\mathrm{H})^{+}$.

\section{General Procedure for the synthesis of compound (5a-1)}

An equimolar mixture of compounds (4a-c) (0.01mol) and appropriate substituted aromatic carboxylic acid hydrazide $(0.01 \mathrm{~mol})$ in phosphorus oxychloride $(20 \mathrm{~mL})$ was refluxion for $6 \mathrm{~h}$. The reaction mixture was cooled to room temperature and then gradually poured on to crushed ice with stirring. The mixture was allowed standing overnight and the solid separated out was filtered, treated with dilute sodium hydroxide solution and washed thoroughly with cold water, dried and recrystallized with DMSO and water to give the title compounds (5a-1) in good yields.

2-(3-(4-Chlorophenyl)-9-methylthieno [3,2-e] $[1,2,4]$ triazolo[4,3-c]pyrimidin-8-yl)-5-(p-tolyl)-1,3,4-oxadiazole $(5 \mathbf{a})$

Yield: 76\% (pale brown color solid); m.p. $214-216^{\circ} \mathrm{C}$; IR (KBr, cm $\left.{ }^{1}\right): 2879,1611,1498,1117 ;{ }^{1} \mathrm{H}$ NMR $\left(400 \mathrm{MHz}\right.$ D DMSO- $\left.d_{6}\right): \delta_{\mathrm{H}} 2.31(\mathrm{~s}$, $\left.3 \mathrm{H}, \mathrm{CH}_{3}\right), 3.02\left(\mathrm{~s}, 3 \mathrm{H}, \mathrm{CH}_{3}\right), 7.19\left(\mathrm{~d}, J_{\mathrm{HH}}=4.0 \mathrm{~Hz}, 2 \mathrm{H}, \mathrm{Ar}-\mathrm{H}\right), 7.50(\mathrm{~d}$, $\left.J_{\mathrm{HH}}=8.0 \mathrm{~Hz}, 2 \mathrm{H}, \mathrm{Ar}-\mathrm{H}\right), 8.17\left(\mathrm{~d}, J_{\mathrm{HH}}=8.0 \mathrm{~Hz}, 2 \mathrm{H}, \mathrm{Ar}-\mathrm{H}\right), 8.47\left(\mathrm{~d}, J_{\mathrm{HH}}=\right.$ $8.0 \mathrm{~Hz}, 2 \mathrm{H}, \mathrm{Ar}-\mathrm{H}), 8.76$ (s, 1H, CH-pyrimidine); ${ }^{13} \mathrm{C}$ NMR (100 MHz; DMSO- $\left.d_{6}\right): \delta_{C} 15.22,23.48,114.57,114.63,114.87,116.97,120.21$, 120.31, 121.14, 125.26, 125.36, 134.36, 139.31, 154.78, 156.76, 157.04, $160.23,161.71,166.59$; LC-MS $(70 \mathrm{eV}): \mathrm{m} / \mathrm{z}=459(\mathrm{M}+\mathrm{H})^{+}$.

2 - ( 4 - Chlorophenyl) - 5 - ( 3 - ( 4 - chlorophenyl) - 9 methylthieno[3,2-e][1,2,4] triazolo $[4,3-c]$ pyrimidin-8-yl)1,3,4-oxadiazole $(5 b)$

2 - ( 4 - chlorophenyl) - 5 - (3- (4 - chlorophenyl) - 9 methylthieno[3,2-e][1,2,4] triazolo[4,3-c]pyrimidin-8-yl)-1,3,4oxadiazoleYield: $73 \%$ (brown color solid); m.p. $234-236^{\circ} \mathrm{C}$; IR ( $\mathrm{KBr}$, $\left.\mathrm{cm}^{-1}\right): 2841,1651,1583,1125 ;{ }^{1} \mathrm{H}$ NMR $\left(400 \mathrm{MHz}\right.$; DMSO- $\left.d_{6}\right): \delta_{\mathrm{H}}$ $2.19\left(\mathrm{~s}, 3 \mathrm{H}, \mathrm{CH}_{3}\right), 6.97\left(\mathrm{~d}, J_{\mathrm{HH}}=8.0 \mathrm{~Hz}, 2 \mathrm{H}, \operatorname{Ar}-\mathrm{H}\right), 7.17\left(\mathrm{~d}, J_{\mathrm{HH}}=\right.$ $12.0 \mathrm{~Hz}, 2 \mathrm{H}, \mathrm{Ar}-\mathrm{H}), 7.71\left(\mathrm{~d}, J_{\mathrm{HH}}=12.0 \mathrm{~Hz}, 2 \mathrm{H}, \mathrm{Ar}-\mathrm{H}\right), 7.91\left(\mathrm{~d}, J_{\mathrm{HH}}\right.$ $=12.0 \mathrm{~Hz}, 2 \mathrm{H}, \mathrm{Ar}-\mathrm{H}), 8.24$ (s, 1H, CH-pyrimidine $) ;{ }^{13} \mathrm{C}$ NMR $(100$ MHz; DMSO- $\left.d_{6}\right): \delta_{C} 15.30,113.61,114.28,117.84,123.57,124.50$, $127.78,128.26,137.54,138.52,142.51,151.24,154.70,156.66$, $163.14,163.71,166.81,169.23$; LC-MS $(70 \mathrm{eV}): \mathrm{m} / \mathrm{z}=479(\mathrm{M}+\mathrm{H})^{+}$. 
2-(3-(4-Chlorophenyl)-9-methylthieno $[3,2-e][1,2,4]$ triazolo[4,3-c]pyrimidin-8-yl)-5-phenyl-1,3,4-oxadiazole $(5 \mathrm{c})$

Yield: $80 \%$ (brown color solid); m.p. $228-230^{\circ} \mathrm{C}$; IR $\left(\mathrm{KBr}, \mathrm{cm}^{-1}\right)$ : 2857, 1663, 1543, 1102; ${ }^{1} \mathrm{H}$ NMR (400 MHz; DMSO- $\left.d_{6}\right): \delta_{\mathrm{H}} 2.07$ (s, $3 \mathrm{H}$, $\left.\mathrm{CH}_{3}\right), 7.20-7.31(\mathrm{~m}, 5 \mathrm{H}, \mathrm{Ar}-\mathrm{H}), 7.45\left(\mathrm{~d}, \mathrm{~J}_{\mathrm{HH}}=12.0 \mathrm{~Hz}, 2 \mathrm{H}, \mathrm{Ar}-\mathrm{H}\right), 7.87$ $\left(\mathrm{d}, J_{\mathrm{HH}}=12.0 \mathrm{~Hz}, 2 \mathrm{H}, \mathrm{Ar}-\mathrm{H}\right), 8.77(\mathrm{~s}, 1 \mathrm{H}, \mathrm{CH}$-pyrimidine $) ;{ }^{13} \mathrm{C} \mathrm{NMR}$ $\left(100 \mathrm{MHz}\right.$; DMSO- $\left.d_{6}\right): \delta_{\mathrm{C}} 16.46,119.40,120.77,123.84,127.33,127.56$, $127.82,136.64,137.16,137.24,140.44,151.71,153.22,157.07,163.66$, 164.60, 169.51, 171.38; LC-MS (70 eV): $\mathrm{m} / \mathrm{z}=445(\mathrm{M}+\mathrm{H})^{+}$.

2-(3-(4-Chlorophenyl)-9-methylthieno $[3,2-\mathrm{e}][1,2,4]$ triazolo[4,3-c]pyrimidin-8-yl)-5-(4-nitrophenyl)-1,3,4oxadiazole $(5 \mathrm{~d})$

Yield: 72\% (brownish yellow color solid); m.p. $253-255^{\circ} \mathrm{C}$; IR $(\mathrm{KBr}$, $\left.\mathrm{cm}^{-1}\right): 2895,1673,1547,1087 ;{ }^{1} \mathrm{H}$ NMR $\left(400 \mathrm{MHz}\right.$; DMSO- $\left.d_{6}\right): \delta_{\mathrm{H}} 1.64$ $\left(\mathrm{s}, 3 \mathrm{H}, \mathrm{CH}_{3}\right), 7.16\left(\mathrm{~d}, J_{\mathrm{HH}}=12.0 \mathrm{~Hz}, 2 \mathrm{H}, \mathrm{Ar}-\mathrm{H}\right), 7.23\left(\mathrm{~d}, J_{\mathrm{HH}}=8.0 \mathrm{~Hz}\right.$, $2 \mathrm{H}, \mathrm{Ar}-\mathrm{H}), 7.68\left(\mathrm{~d}, \mathrm{~J}_{\mathrm{HH}}=8.0 \mathrm{~Hz}, 2 \mathrm{H}, \mathrm{Ar}-\mathrm{H}\right), 7.84\left(\mathrm{~d}, J_{\mathrm{HH}}=12.0 \mathrm{~Hz}, 2 \mathrm{H}\right.$, $\mathrm{Ar}-\mathrm{H}$ ), 8.67 (s, $1 \mathrm{H}, \mathrm{CH}-$ pyrimidine); ${ }^{13} \mathrm{C}$ NMR (100 MHz; DMSO- $d_{6}$ ): $\delta_{\mathrm{C}} 26.64,111.46,119.73,120.90,122.67,126.24,128.05,129.31,130.11$, $137.62,139.20,142.83,144.78,149.68,151.61,157.29,164.34,169.08$; LC-MS $(70 \mathrm{eV}): \mathrm{m} / \mathrm{z}=488(\mathrm{M}-\mathrm{H})$.

2-(4-Chlorophenyl)-5-(9-methyl-3-phenylthieno[3,2-e] $[1,2,4]$ triazolo $[4,3-c]$ pyrimidin-8-yl)-1,3,4-oxadiazole (5e)

Yield: $74 \%$ (yellow color solid); m.p. $219-221^{\circ} \mathrm{C}$; IR $\left(\mathrm{KBr}, \mathrm{cm}^{-1}\right)$ : 2954, 1639, 1577, $1116 ;{ }^{1} \mathrm{H}$ NMR $\left(400 \mathrm{MHz}\right.$; DMSO- $\left.d_{6}\right): \delta_{\mathrm{H}} 1.91(\mathrm{~s}, 3 \mathrm{H}$, $\left.\mathrm{CH}_{3}\right), 7.06-7.28(\mathrm{~m}, 5 \mathrm{H}, \mathrm{Ar}-\mathrm{H}), 7.67\left(\mathrm{~d}, J_{\mathrm{HH}}=8.0 \mathrm{~Hz}, 2 \mathrm{H}, \mathrm{Ar}-\mathrm{H}\right), 7.82$ $\left(\mathrm{d}, J_{\mathrm{HH}}=8.0 \mathrm{~Hz}, 2 \mathrm{H}\right.$, Ar-H), $8.91\left(\mathrm{~s}, 1 \mathrm{H}, \mathrm{CH}\right.$-pyrimidine); ${ }^{13} \mathrm{C} \mathrm{NMR}$ $\left(100 \mathrm{MHz}\right.$; DMSO- $\left.d_{6}\right): \delta_{\mathrm{C}} 15.20,118.62,119.03,121.10,124.84,125.17$, $126.82,129.00,132.37,136.03,138.40,140.66,146.31,151.28,156.09$, 159.00, 163.73, 167.21; LC-MS (70 eV): m/z = $443(\mathrm{M}-\mathrm{H})^{2}$.

2-(9-Methyl-3-phenylthieno[3,2-e][1,2,4] triazolo [4,3-c] pyrimidin-8-yl)-5-phenyl-1,3,4-oxadiazole (5f)

Yield: 68\% (greenish yellow color solid); m.p. 191-193 ${ }^{\circ} \mathrm{C}$; IR $(\mathrm{KBr}$, $\left.\mathrm{cm}^{-1}\right): 2876,1603,1502,1180 ;{ }^{1} \mathrm{H}$ NMR $\left(400 \mathrm{MHz}\right.$; DMSO- $\left.d_{6}\right): \delta_{\mathrm{H}} 2.08$ (s, $3 \mathrm{H}, \mathrm{CH}_{3}$ ), 6.81-6.96 (m, 5H, Ar-H), 7.24-7.36 (m, 5H, Ar-H), 8.27 (s, $1 \mathrm{H}, \mathrm{CH}$-pyrimidine); ${ }^{13} \mathrm{C}$ NMR (100 MHz; DMSO- $\left.d_{6}\right): \delta_{\mathrm{C}} 16.36,113.08$, $120.11,122.19,124.76,126.04,126.91,129.83,131.68,135.79,139.35$, $141.73,144.03,150.61,154.34,160.50,161.62,165.31$; LC-MS (70 eV): $\mathrm{m} / \mathrm{z}=411(\mathrm{M}+\mathrm{H})^{+}$.

2-(9-Methyl-3-phenylthieno[3,2-e] $[1,2,4]$ triazolo $[4,3-c]$ pyrimidin-8-yl)-5-(p-tolyl)-1,3,4-oxadiazole (5g)

Yield: $71 \%$ (brown color solid); m.p. $240-242^{\circ} \mathrm{C}$; IR $(\mathrm{KBr})\left(v_{\max } / \mathrm{cm}\right.$ $\left.{ }^{1}\right): 2816,1644,1584,1166 ;{ }^{1} \mathrm{H}$ NMR $\left(400 \mathrm{MHz}\right.$; DMSO- $\left.d_{6}\right): \delta_{\mathrm{H}} 1.72(\mathrm{~s}$, $\left.3 \mathrm{H}, \mathrm{CH}_{3}\right), 2.23\left(\mathrm{~s}, 3 \mathrm{H}, \mathrm{CH}_{3}\right), 6.76-6.88(\mathrm{~m}, 5 \mathrm{H}, \mathrm{Ar}-\mathrm{H}), 7.42\left(\mathrm{~d}, J_{\mathrm{HH}}=\right.$ $8.0 \mathrm{~Hz}, 2 \mathrm{H}, \mathrm{Ar}-\mathrm{H}), 7.63\left(\mathrm{~d}, J_{\mathrm{HH}}=8.0 \mathrm{~Hz}, 2 \mathrm{H}, \mathrm{Ar}-\mathrm{H}\right), 8.43(\mathrm{~s}, 1 \mathrm{H}, \mathrm{CH}-$ pyrimidine); ${ }^{13} \mathrm{C}$ NMR (100 MHz; DMSO- $\left.d_{6}\right): \delta_{C} 14.15,28.73,108.19$, $119.26,120.35,123.08,125.19,127.37,130.13,133.76,136.28,139.41$, $143.03,149.16,151.70,156.82,161.15,164.02,166.18$; LC-MS (70 eV): $\mathrm{m} / \mathrm{z}=425(\mathrm{M}+\mathrm{H})^{+}$.

2-(9-Methyl-3-phenylthieno[3,2-e] $[1,2,4]$ triazolo [4,3-c] pyrimidin-8-yl)-5-(4-nitrophenyl)-1,3,4-oxadiazole (5h)

Yield: $72 \%$ (brown color solid); m.p. $216-218^{\circ} \mathrm{C}$; IR $\left(\mathrm{KBr}, \mathrm{cm}^{-1}\right)$ : 2815, 1655, 1546, 1005; ${ }^{1} \mathrm{H}$ NMR (400 MHz; DMSO- $\left.d_{6}\right): \delta_{\mathrm{H}} 1.80(\mathrm{~s}, 3 \mathrm{H}$, $\left.\mathrm{CH}_{3}\right), 7.11-7.26(\mathrm{~m}, 5 \mathrm{H}, \mathrm{Ar}-\mathrm{H}), 7.31\left(\mathrm{~d}, \mathrm{~J}_{\mathrm{HH}}=12.0 \mathrm{~Hz}, 2 \mathrm{H}, \mathrm{Ar}-\mathrm{H}\right), 7.70$ $\left(\mathrm{d}, J_{\mathrm{HH}}=8.0 \mathrm{~Hz}, 2 \mathrm{H}, \mathrm{Ar}-\mathrm{H}\right), 8.74(\mathrm{~s}, 1 \mathrm{H}, \mathrm{CH}$-pyrimidine $) ;{ }^{13} \mathrm{C} \mathrm{NMR}$ $\left(100 \mathrm{MHz} ; \mathrm{DMSO}-d_{6}\right): \delta_{\mathrm{C}} 21.94,118.51,121.32,122.96,125.82,127.22$, $128.46,129.71,130.35,134.46,138.22,140.90,143.27,151.10,156.05$, 161.61, 163.30, 167.22; LC-MS (70 eV): $\mathrm{m} / \mathrm{z}=454(\mathrm{M}-\mathrm{H})^{-}$.

2-(4-Chlorophenyl)-5-(9-methyl-3-(p-tolyl)thieno[3,2-e] $[1,2,4]$ triazolo $[4,3-c]$ pyrimidin-8-yl)-1,3,4-oxadiazole $(5 \mathrm{i})$

Yield: 69\% (pale brown color solid); m.p. $187-189^{\circ} \mathrm{C}$; IR (KBr) $\left(v_{\max } / \mathrm{cm}^{-1}\right): 2880,1639,1585,1155 ;{ }^{1} \mathrm{H}$ NMR $\left(400 \mathrm{MHz} ;\right.$ DMSO- $\left.d_{6}\right): \delta_{\mathrm{H}}$ $1.27\left(\mathrm{~s}, 3 \mathrm{H}, \mathrm{CH}_{3}\right), 1.96\left(\mathrm{~s}, 3 \mathrm{H}, \mathrm{CH}_{3}\right), 6.85\left(\mathrm{~d}, J_{\mathrm{HH}}=12.0 \mathrm{~Hz}, 2 \mathrm{H}, \mathrm{Ar}-\mathrm{H}\right)$, $7.16\left(\mathrm{~d}, J_{\mathrm{HH}}=8.0 \mathrm{~Hz}, 2 \mathrm{H}, \mathrm{Ar}-\mathrm{H}\right), 7.29\left(\mathrm{~d}, J_{\mathrm{HH}}=8.0 \mathrm{~Hz}, 2 \mathrm{H}, \mathrm{Ar}-\mathrm{H}\right), 7.82$ $\left(\mathrm{d}, J_{\mathrm{HH}}=8.0 \mathrm{~Hz}, 2 \mathrm{H}, \mathrm{Ar}-\mathrm{H}\right), 8.69$ (s, $1 \mathrm{H}, \mathrm{CH}$-pyrimidine); ${ }^{13} \mathrm{C} \mathrm{NMR}$ $\left(100 \mathrm{MHz}\right.$; DMSO- $\left.d_{6}\right): \delta_{\mathrm{C}} 14.32,21.75,118.19,120.08,124.45,125.09$, $126.96,129.70,131.43,133.00,137.31,139.16,141.45,145.21,148.39$, 153.27, 157.11, 163.08, 166.74; LC-MS (70 eV): m/z $=459(\mathrm{M}+\mathrm{H})^{+}$.

2-(9-Methyl-3-(p-tolyl)thieno[3,2-e] $[1,2,4]$ triazolo[4,3-c] pyrimidin-8-yl)-5-phenyl-1,3,4-oxadiazole $(5 \mathbf{j})$

Yield: $76 \%$ (pale yellow color solid); m.p. $236-238^{\circ} \mathrm{C}$; IR (KBr, cm $\left.{ }^{1}\right): 2824,1602,1502,1180 ;{ }^{1} \mathrm{H}$ NMR $\left(400 \mathrm{MHz} ; \mathrm{DMSO}-d_{6}\right): \delta_{\mathrm{H}} 1.31(\mathrm{~s}$, $\left.3 \mathrm{H}, \mathrm{CH}_{3}\right), 1.82\left(\mathrm{~s}, 3 \mathrm{H}, \mathrm{CH}_{3}\right), 6.89-7.08(\mathrm{~m}, 5 \mathrm{H}, \mathrm{Ar}-\mathrm{H}), 7.38\left(\mathrm{~d}, \mathrm{~J}_{\mathrm{HH}}=\right.$ $8.0 \mathrm{~Hz}, 2 \mathrm{H}, \mathrm{Ar}-\mathrm{H}), 7.58\left(\mathrm{~d}, J_{\mathrm{HH}}=8.0 \mathrm{~Hz}, 2 \mathrm{H}, \mathrm{Ar}-\mathrm{H}\right), 8.81(\mathrm{~s}, 1 \mathrm{H}, \mathrm{CH}-$ pyrimidine); ${ }^{13} \mathrm{C}$ NMR (100 MHz; DMSO- $\left.d_{6}\right): \delta_{\mathrm{C}} 11.41,29.62,119.48$, $122.71,124.01,125.66,127.10,129.34,132.25,135.14,137.23,139.47$, $140.13,144.16,151.81,155.05,159.00,160.36,164.40$; LC-MS $(70 \mathrm{eV})$ : $\mathrm{m} / \mathrm{z}=425(\mathrm{M}+\mathrm{H})^{+}$

2-(9-Methyl-3-(p-tolyl)thieno[3,2-e] $[1,2,4]$ triazolo[4,3-c] pyrimidin-8-yl)-5-(p-tolyl)-1,3,4-oxadiazole (5k)

Yield: 83\% (brown color solid); m.p. $196-198^{\circ} \mathrm{C}$; IR $\left(\mathrm{KBr}, \mathrm{cm}^{-1}\right)$ : 2846, 1628, 1548, 1132; ${ }^{1} \mathrm{H}$ NMR (400 MHz; DMSO- $d_{6}: \delta_{\mathrm{H}} 1.42(\mathrm{~s}, 3 \mathrm{H}$, $\left.\mathrm{CH}_{3}\right), 2.31\left(\mathrm{~s}, 6 \mathrm{H}, \mathrm{CH}_{3}\right), 7.16\left(\mathrm{~d}, J_{\mathrm{HH}}=12.0 \mathrm{~Hz}, 2 \mathrm{H}, \mathrm{Ar}-\mathrm{H}\right), 7.26\left(\mathrm{~d}, J_{\mathrm{HH}}\right.$ $=8.0 \mathrm{~Hz}, 2 \mathrm{H}, \mathrm{Ar}-\mathrm{H}), 7.67\left(\mathrm{~d}, J_{\mathrm{HH}}=8.0 \mathrm{~Hz}, 2 \mathrm{H}, \mathrm{Ar}-\mathrm{H}\right), 7.83\left(\mathrm{~d}, J_{\mathrm{HH}}=\right.$ $8.0 \mathrm{~Hz}, 2 \mathrm{H}, \mathrm{Ar}-\mathrm{H}), 8.92$ (s, 1H, CH-pyrimidine); ${ }^{13} \mathrm{C}$ NMR $(100 \mathrm{MHz}$ DMSO $\left.-d_{6}\right): \delta_{\mathrm{C}} 16.02,30.12,120.48,123.91,124.32,125.20,128.55$, $129.34,131.06,134.10,136.68,139.19,141.28,148.57,150.33,156.45$, $160.07,162.10,165.86$; LC-MS $(70 \mathrm{eV}): \mathrm{m} / \mathrm{z}=437(\mathrm{M}-\mathrm{H})$.

2-(9-Methyl-3-(p-tolyl)thieno[3,2-e] $[1,2,4]$ triazolo[4,3-c] pyrimidin-8-yl)-5-(4-nitro pheny 1$)-1,3,4$-oxadiazole (5l)

Yield: $71 \%$ (yellow color solid); m.p. $212-214^{\circ} \mathrm{C}$; IR $\left(\mathrm{KBr}, \mathrm{cm}^{-1}\right)$ : $2836,1624,1543,1150 ;{ }^{1} \mathrm{H}$ NMR $\left(400 \mathrm{MHz}\right.$; DMSO- $\left.d_{6}\right): \delta_{\mathrm{H}} 1.17(\mathrm{~s}, 3 \mathrm{H}$, $\left.\mathrm{CH}_{3}\right), 2.26\left(\mathrm{~s}, 3 \mathrm{H}, \mathrm{CH}_{3}\right), 6.66\left(\mathrm{~d}, J_{\mathrm{HH}}=8.0 \mathrm{~Hz}, 2 \mathrm{H}, \mathrm{Ar}-\mathrm{H}\right), 7.42\left(\mathrm{~d}, \mathrm{~J}_{\mathrm{HH}}\right.$ $=12.0 \mathrm{~Hz}, 2 \mathrm{H}, \mathrm{Ar}-\mathrm{H}), 7.57\left(\mathrm{~d}, J_{\mathrm{HH}}=12.0 \mathrm{~Hz}, 2 \mathrm{H}, \mathrm{Ar}-\mathrm{H}\right), 8.04\left(\mathrm{~d}, J_{\mathrm{HH}}=\right.$ $8.0 \mathrm{~Hz}, 2 \mathrm{H}, \mathrm{Ar}-\mathrm{H}), 8.36$ (s, 1H, CH-pyrimidine); ${ }^{13} \mathrm{C}$ NMR $(100 \mathrm{MHz}$; DMSO- $\left.d_{6}\right): \delta_{\mathrm{C}} 13.49,16.51,103.38,103.73,104.05,140.42,110.46$, $110.75,119.69,124.42,128.40,128.52,141.47,153.83,154.65,157.65$, $157.80,162.64,171.24 ; \mathrm{LC}-\mathrm{MS}(70 \mathrm{eV}): \mathrm{m} / \mathrm{z}=470(\mathrm{M}+\mathrm{H})^{+}$.

\section{Biological Assay}

\section{Antibacterial studies}

The newly prepared compounds were screened for their antibacterial activity against Bacillus subtilis, Staphylocouccus aureus, Klebsiella pneumonia and Escherichia coli (clinical isolate) bacterial strains by disc diffusion method $[24,25]$. A standard inoculums $\left(1-2 \times 10^{7}\right.$ c.f.u. $/ \mathrm{ml}$ $0.5 \mathrm{McF}$ arland standards) were introduced on to the surface of sterile agar plates, and a sterile glass spreader was used for even distribution of the inoculums. The disks measuring $6 \mathrm{mmin}$ diameters were prepared from Whatman no. 1 filter paper and sterilized by dry heat at $140^{\circ} \mathrm{C}$ for $1 \mathrm{~h}$. The sterile disks previously soaked in a known concentration of 
the test compounds were placed in nutrient agar medium. Solvent and growth controls were kept. Amoxicillin $(30 \mu \mathrm{g})$ was used as positive control and the disk poured in DMSO was used as negative control and the test compounds were dissolved in DMSO at concentration of 100 and $50 \mu \mathrm{g} / \mathrm{mL}$. The plates were inverted and incubated for 24 $\mathrm{h}$ at $37^{\circ} \mathrm{C}$. The susceptibility was assessed on the basis of diameter of zone of inhibition against Gram-positive and Gram-negative strains of bacteria. Inhibition of zone of measured and compared with controls. The bacterial zone of inhibition values are given in (Table 1).

\section{Antifungal studies}

The newly prepared compounds were screened for their antifungal activity against Candida albicans and Aspergillus flavus in DMSO by agar diffusion method [26]. Sabourauds agar media was prepare3d by dissolving peptone ( $1 \mathrm{~g})$, D-glucose $(4 \mathrm{~g})$ and agar $(2 \mathrm{~g})$ in distilled water $(100 \mathrm{ml})$ and adjusting $\mathrm{pH}$ 5.7. Normal saline was used to make suspension of corresponding species. Twenty millilitres of agar media was poured into each Petri dish. Excess of suspension was decanted and the plates were dried by placing in an incubator at $37^{\circ} \mathrm{C}$ for $1 \mathrm{~h}$ using an agar punch, wells were made and each well was labelled. A control was also prepared in triplicate and maintained at $37^{\circ} \mathrm{C}$ for $3-4$ days. The fungal activity of each compound was compared with Ketoconazole as a standard drug. Inhibition zone were measured and compared with the controls. The fungal zone of inhibition values are given in (Table 2).

\section{Result and Discussion}

\section{Chemistry}

The reaction sequences employed for synthesis of title compounds are shown in (Scheme 1). In the present work, the starting ethyl 5-amino-4-cyano-3-methylthiophene-2-carboxylate (1) was prepared according to Gewald synthetic procedure [21]. The ethyl thieno[1,2,4] triazolopyrimidine carboxylate $(3 a-c)$ were synthesized from ethyl 4-cyano-5-((ethoxymethylene)amino)-3-methylthiophene-2carboxylate (2) with substituted aroylhydrazides in toluene at refluxion temperature, which on further treatment with $3 \mathrm{a}-\mathrm{c}$ in methanolic sodium hydroxide gave $4 \mathrm{a}-\mathrm{c}$. Further, the triazole carboxylic acids (4ac) were treated with substituted aroylhydrazides in $\mathrm{POCl}_{3}$ and afforded 1,3,4-oxadiazoles (5a-1). All compounds displayed IR, ${ }^{1} \mathrm{H}$ and ${ }^{13} \mathrm{C}$ NMR and mass spectra consistent with the assigned structures. ${ }^{1} \mathrm{H}$ NMR and IR spectrum of compounds ( $3 \mathrm{a}-\mathrm{c}$ ) showed triplet at 1.36-1.44 and a quartet at 4.42-4.44 ppm are due to the carboxylic ethyl ester and the most characteristic absorption bands are at $1705-1716 \mathrm{~cm}^{-1}(\mathrm{C}=\mathrm{O})$ and 1524$1562 \mathrm{~cm}^{-1}(\mathrm{C}=\mathrm{N})$ groups. When compounds $(3 \mathrm{a}-\mathrm{c})$ were converted to

\begin{tabular}{|c|c|c|c|c|c|c|c|c|}
\hline \multicolumn{9}{|c|}{ Zone of inhibition measure in $\mathrm{mm}$} \\
\hline \multirow{3}{*}{$\begin{array}{l}\text { Synthesized } \\
\text { compounds }\end{array}$} & \multicolumn{4}{|c|}{ Gram positive } & \multicolumn{4}{|c|}{ Gram negative } \\
\hline & \multicolumn{2}{|c|}{ Bacillus subtilis } & \multicolumn{2}{|c|}{ Staphylocouccus aureus } & \multicolumn{2}{|c|}{ Klebsiella pneumonia } & \multicolumn{2}{|c|}{ Escherichia coli } \\
\hline & $100 \mu \mathrm{g} / \mathrm{mL}$ & $50 \mu \mathrm{g} / \mathrm{mL}$ & $100 \mu \mathrm{g} / \mathrm{mL}$ & $50 \mu \mathrm{g} / \mathrm{mL}$ & $100 \mu \mathrm{g} / \mathrm{mL}$ & $50 \mu \mathrm{g} / \mathrm{mL}$ & $100 \mu \mathrm{g} / \mathrm{mL}$ & $50 \mu \mathrm{g} / \mathrm{mL}$ \\
\hline $5 a$ & 10.5 & 7.5 & 10.5 & 8.0 & 11.5 & 9.5 & 12.0 & 9.0 \\
\hline $5 b$ & 12.5 & 10.0 & 14.5 & 10.5 & 15.0 & 13.5 & 16.5 & 12.5 \\
\hline $5 c$ & 11.0 & 9.5 & 11.5 & 8.5 & 12.5 & 12.0 & 13.0 & 11.5 \\
\hline $5 d$ & 13.0 & 10.5 & 15.0 & 11.5 & 16.5 & 14.0 & 17.0 & 13.0 \\
\hline $5 e$ & 10.0 & 8.0 & 11.0 & 9.5 & 13.0 & 11.0 & 13.5 & 11.0 \\
\hline $5 f$ & 8.5 & 6.5 & 9.0 & 6.5 & 10.5 & 8.0 & 11.0 & 8.0 \\
\hline $5 g$ & 7.0 & 4.5 & 7.0 & 4.5 & 8.5 & 6.5 & 9.0 & 7.0 \\
\hline $5 \mathrm{~h}$ & 11.5 & 9.0 & 12.5 & 11.0 & 14.5 & 11.5 & 15.5 & 12.0 \\
\hline $5 i$ & 9.5 & 7.0 & 9.5 & 7.5 & 12.0 & 10.0 & 12.5 & 10.5 \\
\hline $5 j$ & 7.5 & 3.5 & 8.0 & 7.0 & 9.5 & 7.0 & 10.5 & 7.5 \\
\hline $5 k$ & 6.0 & 3.0 & 7.5 & 5.0 & 8.0 & 6.0 & 9.5 & 6.0 \\
\hline 51 & 10.5 & 8.5 & 10.0 & 9.0 & 13.5 & 10.5 & 14.0 & 11.0 \\
\hline Amoxicillin & 15.5 & 12.5 & 17.5 & 13.0 & 18.0 & 14.5 & 19.5 & 15.5 \\
\hline Control (DMSO) & - & - & - & - & - & - & - & - \\
\hline
\end{tabular}

Table 1: Antibacterial activity of compounds 5a-l.

\begin{tabular}{|c|c|c|c|c|}
\hline \multicolumn{5}{|c|}{ Zone of inhibition measure in $\mathrm{mm}$} \\
\hline \multirow{2}{*}{ Synthesized compounds } & \multicolumn{2}{|c|}{ Candida albicans } & \multicolumn{2}{|c|}{ Aspergillus flavus } \\
\hline & $100 \mu \mathrm{g} / \mathrm{mL}$ & $50 \mu \mathrm{g} / \mathrm{mL}$ & $100 \mu \mathrm{g} / \mathrm{mL}$ & $50 \mu \mathrm{g} / \mathrm{mL}$ \\
\hline $5 a$ & 10.5 & 9.5 & 9.5 & 7.0 \\
\hline $5 b$ & 14.5 & 12.0 & 12.5 & 9.5 \\
\hline $5 c$ & 13.0 & 11.5 & 10.5 & 8.0 \\
\hline $5 d$ & 17.5 & 12.5 & 16.0 & 12.0 \\
\hline $5 e$ & 11.0 & 9.0 & 10.0 & 9.0 \\
\hline $5 f$ & 9.5 & 7.5 & 8.0 & 6.5 \\
\hline $5 g$ & 8.0 & 5.5 & 7.0 & 3.5 \\
\hline $5 \mathrm{~h}$ & 12.5 & 8.0 & 10.5 & 10.0 \\
\hline $5 i$ & 8.5 & 5.0 & 7.5 & 5.5 \\
\hline $5 j$ & 6.5 & 4.5 & 7.0 & 4.0 \\
\hline $5 \mathrm{k}$ & 5.0 & 3.0 & 4.5 & 2.0 \\
\hline 51 & 11.5 & 6.5 & 9.0 & 6.0 \\
\hline Ketoconazole & 20.5 & 16.0 & 18.5 & 14.0 \\
\hline Control (DMSO) & - & - & - & - \\
\hline
\end{tabular}

Table 2: Antifungal activity of compounds 5a-l. 


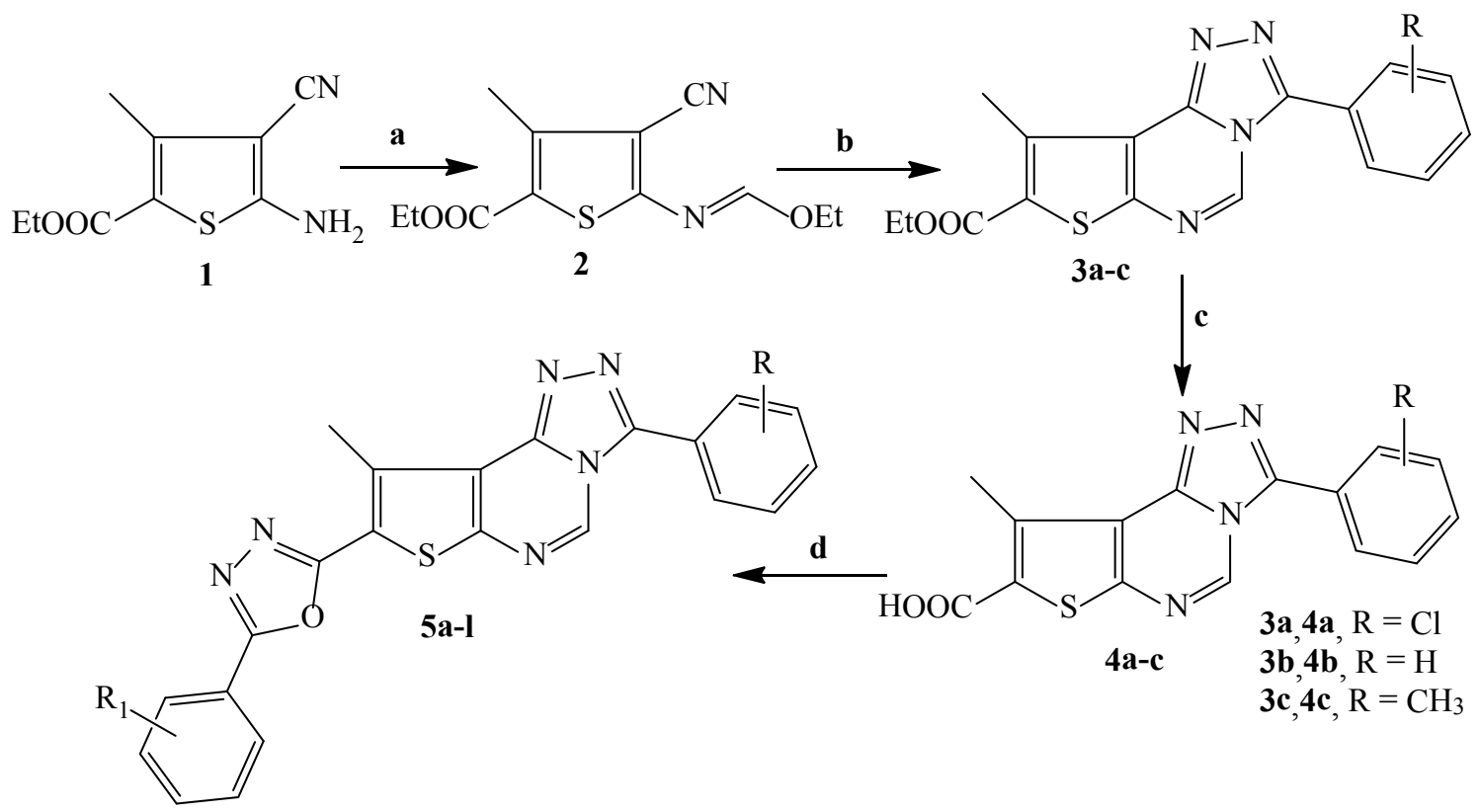

\begin{tabular}{|l|l|l|l|l|l|l|l|l|l|l|l|l|l|l|l}
\hline 5 & a & b & c & d & e & f & g & $h$ & l & l \\
\hline $\mathrm{R}$ & $4-\mathrm{Cl}$ & $4-\mathrm{Cl}$ & $4-\mathrm{Cl}$ & $4-\mathrm{Cl}$ & $4-\mathrm{H}$ & $4-\mathrm{H}$ & $4-\mathrm{H}$ & $4-\mathrm{H}$ & $4-\mathrm{CH}_{3}$ & $4-\mathrm{CH}_{3}$ & $4-\mathrm{CH}_{3}$ & $4-\mathrm{CH}_{3}$ \\
\hline $\mathrm{R}_{1}$ & $4-\mathrm{CH}_{3}$ & $4-\mathrm{Cl}$ & $4-\mathrm{H}$ & $4-\mathrm{NO}_{2}$ & $4-\mathrm{Cl}$ & $4-\mathrm{H}$ & $4-\mathrm{CH}_{3}$ & $4-\mathrm{NO}_{2}$ & $4-\mathrm{Cl}$ & $4-\mathrm{H}$ & $4-\mathrm{CH}_{3}$ & $4-\mathrm{NO}_{2}$ \\
\hline
\end{tabular}

Reagents and Conditions: (a) Triethyl orthoformate, toluene, reflux; (b) R-PhCONHNH${ }_{2}$, toluene, $\mathrm{AcOH}, \mathrm{reflux}, 12 \mathrm{~h}$; (c) $\mathrm{MeOH}, \mathrm{NaOH}$, rt; (d) Substituted acid hydrazides, $\mathrm{POCl}_{3}$, reflux, $6 \mathrm{~h}$.

Scheme 1: Synthetic path way of preparation of 1,3,4-oxadiazole and 1,2,4-triazolethienopyrimidine derivatives.

carboxylic acids of 4-substituted phenyl-9-methyl-thieno[3,2-e][1,2,4] triazolo[4,3-c]pyrimidine in basic media, carboxylic ethyl ester peaks were disappeared, while new signals were observed due to the $\mathrm{COOH}$ group at 10.14-10.62 $\mathrm{ppm}$ in the ${ }^{1} \mathrm{H}$ NMR and IR spectrum the most characteristic absorption bands are at $3319-3364 \mathrm{~cm}^{-1}(\mathrm{OH}), 1702-1714$ $\mathrm{cm}^{-1}(\mathrm{C}=\mathrm{O})$ and $1512-1542 \mathrm{~cm}^{-1}(\mathrm{C}=\mathrm{N})$ groups of compounds $(4 \mathrm{a}-\mathrm{c})$. Compounds (5a-1) were partly characterised by the absorption bands at $1005-1180 \mathrm{~cm}^{-1}$ and $1498-1585 \mathrm{~cm}^{-1}$ in IR spectra due to $\mathrm{C}-\mathrm{O}-\mathrm{C}$ and $\mathrm{C}=\mathrm{N}$ groups respectively. Its ${ }^{1} \mathrm{H} \mathrm{NMR}$ spectra showed a singlet at 1.17$2.31 \mathrm{ppm}$ due to the thiophene methyl group and another singlet at 8.27-8.92 ppm due to proton of pyrimidine moiety. All other aromatic protons appeared in 6.60-8.04 ppm regions. The mass spectra of all the final derivatives showed comparable molecular ion peak with respect to molecular formula.

\section{Antimicrobial studies}

The newly synthesized compounds (5a-1) were screened for their in-vitro antibacterial activity against Bacillus subtilis, Staphylocouccus aureus, Klebsiella pneumonia and Escherichia coli using Amoxicillin as standard by disc diffusion method (zone of inhibition) [24,25]. The test compounds were dissolved in dimethylsulfoxide (DMSO) at concentrations of 50 and $100 \mu \mathrm{g} / \mathrm{mL}$. The antibacterial screening revealed that all the tested compounds showed good inhibition against various tested microbial strains compared to the standard drug. Along with the synthesized compounds $5 b, 5 c, 5 d$ and $5 \mathrm{~h}$ were found to be more active against tested bacterial strains as compared to the standard. The enhanced antibacterial activity of $5 b, 5 c$ and $5 d$ were due to presence of chlorine in the 1,2,4-triazole at the fourth position of thienopyrimidine moiety. The compound $5 \mathrm{~h}$ contains nitro group at fourth position of 1,3,4-oxadiazole of thienopyrimidine ring which accounts for the enhanced antibacterial activity. Compound $5 \mathrm{e}$ exhibited moderate antibacterial activity against all tested bacterial stains. In general, increase of electron donating strength on the 1,2,4-triazole and 1,3,4-oxadiazole (methyl substitution) decreases antibacterial activity. On the other hand, introducing halogen or electron withdrawing phenyl ring on the 1,2,4-triazole and 1,3,4-oxadiazole with thienopyrimidine increases the antibacterial activity. The activity exhibited by the synthesized compounds were due to both 1,2,4-triazole and 1,3,4-oxadiazole core rings (Scheme 2).

The in-vitro antifungal activities for compounds 5a-1 were determined by agar diffusion method [26]. The results indicate that, among the tested compounds $5 \mathrm{~b}$ and $5 \mathrm{~d}$ were active against all tested fungal strains. The enhanced activities are due to electron withdrawing groups viz., chloro and nitro attached to heterocyclic moieties (1,2,4-triazole and 1,3,4-oxadiazole) of thienopyrimidine ring. All other compounds such as, 1,2,4-triazole and 1,3,4-oxadiazole with methyl and phenyl substitution with thienopyrimidine showed lesser antifungal activity as compared with standard Ketoconazole. The (Tables 1 and 2) depict the antimicrobial screening results of the final compounds.

\section{Conclusion}

The research study reports the successful synthesis and antimicrobial activity of 1,2,4-triazole and 1,3,4-oxadiazole bearing thienopyrimidine moiety. The antimicrobial activity study revealed that all the tested compounds showed good antibacterial and antifungal activities against pathogenic strains. The structure and biological activity relationship of title compounds indicate that the presence of electron withdrawing groups like chloro and nitro groups attached to the triazole and oxadiazole rings were responsible for good antimicrobial activity and hence compounds $5 \mathrm{~b}$ and $5 \mathrm{~d}$ exhibited more potent antimicrobial activity of all tested pathogenic straines. 
<smiles>[R]C(=O)NNCCCC(CC)=Nc1sccc1C#N</smiles><smiles>[R]C(=O)NNC(=N)c1ccsc1NC=O</smiles><smiles>[R]c1nnc2c3ccsc3ncn12</smiles>

Scheme 2: A plausible mechanism pathway for the formation of 1,2,4-triazole.

\section{Acknowledgement}

The authors would like to express their gratitude and thanks to the Council of Scientific and Indusrial Research (CSIR), New Delhi, for financial assistance under major research project CSIR Lr. No.02 (0065)/12/EMR-II.

\section{References}

1. Gilks CF (1998) Acute bacterial infections and HIV disease. Brit Med Bull 54 383-393.

2. Samaras V, Rafailidis PI, Mourtzoukou EG, Peppas G, Falagas ME (2010) Chronic bacterial and parasitic infections and cancer. J Infect Dev Ctries 4: 267-281.

3. Wang XL, Wan K, Zhou CH (2010) Synthesis of novel sulfanilamide derived 1,2,3-triazoles and their evaluation for antibacterial and antifungal activities. Eur J Med Chem 45: 4631-4639.

4. Bremner JB, Ambrus JI, Samosorn S (2007) Dual action-based approaches to antibacterial agents. Curr Med Chem 14: 1459-1477.

5. Hubschwerlen C, Specklin JL, Sigwalt C, Schroeder S, Locher HH (2003) Design, synthesis and biological evaluation of oxazolidinone-quinolone hybrid. Bioorg Med Chem 11: 2313-2319.

6. Nasr MN, Gineinah MM (2002) Pyrido[2,3-d]pyrimidines and pyrimido [5,4$5,6]$ pyrido [2,3-d]pyrimidines as new antiviral agents, synthesis and biological activity. Arch Pharm Pharm Med Chem 335: 289-295.

7. Chambhare RV, Khadse BG, Bobde AS, Bahekar RH (2003) Synthesis and preliminary evalution of some $\mathrm{n}$-[5-(2-furanyl)-2-methyl-4-oxo-4h-thieno[2,3-d] pyrimidine-3-yl]-carboxamide and 3-substituted-5-(2-furanyl)-2-methyl-3hthieno[2,3-d]pyrimidine -4-ones as antimicrobial agents. Eur J Med Chem 38: 89-100.

8. Russell RK, Press JB, Rampulla RA, McNally JJ, Falotico R, et al. (1988) Thienopyrimidinedione derivatives as potential antihypertensive agents. J Med Chem 31: 1786-1793.

9. Alagarsamy V, Meena S, Ramseshu KV, Solomon VR, Thirumurugan K et al. (2006) Synthesis, analgesic, anti-inflammatory, ulcerogenic index and antibacterial activities of novel 2-methylthio-3-substituted-5,6,7,8- tetrahydrobenzo thieno[2,3-d]pyrimidin-4(3h)-ones. Eur J Med Chem 41: 1293 1300.

10. Petrie CR, Cottam HB, Mckernan PA, Robins RK, Revankar GR (1985) Synthesis and biological activity of 6-azacadeguomycin and certain 3,4,6-trisubstituted pyrazolo [3,4-d]pyrimidine ribonuleosi. J Med Chem 28: 1010-1016.

11. Dolman SJ, Gosselin F, Oshea PD, Davies IW (2006) Superior reactivity of thiosemi carbazides in the synthesis of 2-amino-1,3,4-oxadiazoles. J Org Chem 71: 9548-9551.

12. Mullican MD, Wilson MW, Connor DT, Kostlan CR, Schrier DJ, et al. (1993) Design of 5-(3,5-di-tert-butyl-4-hydroxyphenyl)-I,3,4-thiadiazoles1,3,4-oxadiazoles, and 1,2,4-triazoles as orally-active, nonulcerogenic antiinflammatory agents. J Med Chem 36: 1090-1099.

13. Shi W, Qian X, Zhang R, Song G (2001) Synthesis and quantitative structureactivity relationships of new 2,5-disubstituted-1,3,4-oxadiazoles. J Agric Food Chem 49: 124-130.

14. Gaonkar SL, Rai KML, Prabhuswamy B (2006) Synthesis and antimicrobia studies of a new series of 2-\{4-[2-(5-ethylpyridin-2-yl)ethoxy]phenyl\}-5substituted-1,3,4-oxadiazoles. Eur J Med Chem 41: 841-846.

15. Patel RV, Pate PK, Kumari P, Rajani DP, Chikhalia KH (2012) Synthesis of benzimida zolyl-1,3,4-oxadiazol-2ylthio-n-phenyl (benzothiazolyl) acetamides as antibacterial, antifungal and antituberculosis agents. Eur J Med Chem 53 $41-51$.

16. Thompson GR, Cadena J, Patterson TF (2009) Overview of antifungal agents. Clin Chest Med 30: 203-215.

17. Gill C, Jadhav G, Shaikh M, Kale R, Ghawalkar A, et al. (2008) Clubbed [1,2,3] triazoles by fluorine benzimidazole a novel approach to H37Rv inhibitors as a potential treatment for tuberculosis. Bioorg Med Chem Lett 18: 6244-6247.

18. Kamal A, Shankaraiah N, Devaiah V, Reddy KL, Juvekar A, et al. (2008) Synthesis of 1,2,3-triazole-linked pyrrolobenzodiazepine conjugates employing click chemistry, DNA-binding anity and anticancer activity. Bioorg Med Chem Lett 18: 1468-1473.

19. Kotaiah Y, Harikrishna N, Nagaraju K, Rao CV (2012) Synthesis and antioxidant activity of 1,3,4-oxadiazole tagged thieno[2,3-d] pyrimidine derivatives. Eur $J$ Med Chem 58: 340-345. 
Citation: Kerru N, Settypalli T, Nallapaneni H, Chunduri VR (2014) Novel Thienopyrimidine Derivatives Containing 1,2,4-triazoles and 1,3,4-oxadiazoles as Potent Antimicrobial Activity. Med chem 4: 623-629. doi:10.4172/2161-0444.1000204

20. Nagaraju K, Kotaiah Y, Sampath C, Harikrishna N, Rao CV (2013) A facile synthesis of some novel fused $[1,2,4]$ triazolo[3,4-b][1,3,4]thiadiazol derivatives. J Sulfur Chem 34: 264-275.

21. Huang XG, Liu J, Ren J, Wang T, Chen W, et al. (2011) A facile and practical one-pot synthesis of mutisubstituted 2-aminothiophenes via imidazolecatalyzed Gewald reaction. Tetrahedron 67: 6202-6205.

22. Shridhar IP, Jameel ASM, Nitinkumar SS, Mohammed IAK, Ashraf YK, et al. (2011) Benzothieno[3,2-e][1,2,4]triazolo[4,3-c]pyrimidines synthesis characterization antimicrobial activity and incorporation into solid liquid nanoparticles. Arch Pharm Chem Life Sci 11: 358-365.
23. Panchamukhi SI, Mohammed IAK, Khan AY, Kalashetti MB, Khazi IM (2010) Synthesis characterization antibacterial and antifungal activity of thienopyrimidines and triazolothienopyrimidines. Pharmaceutical Chemistry Journal 44: 694-696.

24. Cruickshank R, Duguid JP, Marmion BP, Swain RHA (1975) In Medicinal microbiology. (12) Churchill Livingstone, London.

25. Collins AH (1976) Microbiological methods (2) Butterworth, London.

26. Varma RS (1998) Antifungal agents: past, present and future prospects, National Academy of Chemistry \& Biology, Lucknow, India. 